\title{
Ventajas e inconvenientes de la encuesta por Internet ${ }^{1}$
}

\author{
Vidal Díaz de Rada \\ Universidad Pública de Navarra. Departamento de Sociología \\ vidal@unavarra.es
}

Recibido: 02-02-2010

Aceptado: 31-05-2011

\section{Resumen}

En este trabajo, se comparan las encuestas autoadministradas a través de Internet con las presenciales y telefónicas, destacando las ventajas y los inconvenientes de cada una. La encuesta por Internet tiene, entre sus grandes virtudes, la gran rapidez, la mejora en la respuesta por la posibilidad de introducir elementos audiovisuales en el cuestionario y el menor coste de la investigación cuando se compara con encuestas administradas. Entre sus grandes inconvenientes, encontramos los errores de cobertura, la ausencia de aleatoriedad de las muestras y la menor tasa de respuesta.

Existen importantes diferencias entre la población general y los usuarios de Internet, puesto que las encuestas a través de la red presentan un perfil más joven y con mayor nivel educativo. La práctica totalidad de las investigaciones analizadas localizan también diferencias en las variables objeto de la investigación, según se haya respondido el cuestionario frente a un entrevistador o en el ordenador del entrevistado.

En definitiva, la revisión de la literatura sobre el tema sugiere prudencia a la hora de aceptar que los resultados de la aplicación de un cuestionario sean los mismos, con independencia del modo de aplicación utilizado.

Palabras clave: encuesta por Internet; encuesta en línea; efectos producidos por el modo de recogida de información; encuesta presencial.

1. Este texto surge tras la revisión de un trabajo enviado para ser publicado en una revista especializada. La cantidad de ausencias sobre las condiciones más adecuadas - e inadecuadas - donde utilizar las encuestas por Internet, unida a la escasez de trabajos sobre el tema publicados en nuestro idioma, me llevaron a escribir este estudio, donde se sintetizan las ventajas y los inconvenientes de estas encuestas.

El autor desea agradecer las sugerencias recibidas por tres revisores anónimos, así como por el consejo editorial de PAPERS. Gracias al Instituto de Estudios Avanzados del CSIC (Córdoba), por permitir la utilización del estudio Encuesta sobre la situación de los andaluces en el extranjero (financiado por la Consejería de Gobernación de la Junta de Andalucía). Gracias también a Juan Antonio Domínguez Álvarez, por la preparación de un archivo específico para analizar la influencia de las preguntas abiertas. 


\section{Abstract: Advantages and disadvantages of Internet research surveys}

The aim of this paper is to analyze whether the results of an internet survey are similar to faceto-face, telephone and mail surveys. The analysis of the research reveals that the people who answer the internet survey are younger and better-educated, making it impossible to accept the first part of the hypothesis. Most of the research about this topic show differences between modes in attitude questions. Those who respond to the face-to-face surveys provide a more positive ideas about the use of the survey, as well as greater confidence in their results. However, people who responded to the questionnaire on the Internet believe that more respondents answered with total honesty, carry out better monitoring of results of surveys on political, social and cultural rights, as well as a higher valuation on the usefulness of the surveys.

Key words: web surveys; online surveys; survey mode effects; face-to-face survey.

\section{Sumario}

1. Delimitación conceptual y terminológica: encuestas autoadministradas

2. Ventajas e inconvenientes de las diferentes modalidades de encuesta
3. Comparativa entre modalidades

de recogida de información

4. Conclusiones

Referencias bibliográficas

Anexo. Características técnicas de la investigación

La emergencia de las encuestas por Internet es consecuencia del gran desarrollo experimentado en las modalidades ${ }^{2}$ de recogida de información que tuvo lugar en los últimos veinte años del siglo XX (Tourangeau, 2004). La elevada utilización de la encuesta telefónica en las décadas de 1960 y 1970, generada fundamentalmente por el encarecimiento de la recogida de información con encuesta presencial, fue decisiva para el desarrollo de sistemas automatizados de recogida de información (Computer Assisted Telephone Interview-CATI) en las décadas de 1970 y 1980. Los grandes avances informáticos experimentados en la última década del citado siglo — por la reducción del tamaño y el peso de los equipos, así como el aumento de la autonomía de las baterías- generó un gran desarrollo de la encuesta presencial asistida con ordenador (Computer Assisted Personal Interview-CAPI) en la última década del siglo XX (Kalton, 2000; Tourangeau, 2004; Schräpler et al., 2010).

En el desarrollo de las encuestas sin entrevistador (autoadministradas), debe considerarse, por un lado, su elevado uso, debido al gran aumento de la no respuesta en la cooperación en las encuestas presenciales y telefónicas en las últimas décadas del siglo XX (Dillman, 1991) y, por otro, las enormes posibilidades que ofrece Internet para realizar la distribución y el reenvío de un gran

2. Methods ha sido el término utilizado en el ámbito anglosajón para definir las diferentes herramientas de recogida de información. Nos parece más correcta la traducción por procedimientos, modos o modalidades, esta última palabra será la que emplearemos en el presente trabajo. 
número de cuestionarios con gran rapidez (Fox et al., 2003), precisamente uno de los grandes inconvenientes de las encuestas por correo (Couper, 2000; Dillman, 2000; Dillman et al., 2009).

De hecho, en la actualidad, se está produciendo un gran aumento en la utilización de la encuesta por Internet, lo cual está motivado, sin duda, por sus características intrínsecas, como son la rapidez en la recogida de información, el bajo coste, la mejora en las respuestas y otros factores que se presentarán más adelante. Son características que llevan a poner en duda las tradicionales ventajas de la encuesta presencial; modalidad considerada, hasta hace pocos años, como la mejor forma de recogida de información (De Leeuw y Van der Zouwen, 1988; Biemer y Lyberg, 2003; Groves et al., 2009; De Leeuw, 2008; Dykema et al., 2008). El informe sobre la visión global del mercado de la investigación en 2009 — realizada en veintidós países - da cuenta del crecimiento de las encuestas por Internet cuando señalan que el $21 \%$ de la investigación realizada durante 2009 se llevó a cabo en línea, frente al 18\% realizada a través del teléfono y el $12 \%$ cara a cara ${ }^{3}$ (Cuende, 2010). Otros expertos señalan que el $20 \%$ de la investigación que se realiza en Europa es en línea y que en USA, Japón y Canadá alcanza el 35\% (Martín y Moré, 2010).

El fin de este trabajo es analizar si los resultados obtenidos por encuestas autoadministradas a través de Internet son equivalentes a los recogidos con encuestas presenciales y telefónicas, realizando una revisión bibliográfica de la literatura sobre el tema. El objetivo es comprobar si existe equivalencia entre modalidades, tanto en el tipo de población que coopera en cada modalidad, como en las preguntas de la encuesta.

\section{Delimitación conceptual y terminológica: encuestas autoadministradas}

El aspecto básico que caracteriza a la encuesta autoadministrada es la ausencia de un entrevistador que lea las preguntas y anote las respuestas. Estas tareas recaen en la persona que responde al cuestionario (Erdos, 1970; De Leeuw, 1992). Las encuestas autoadministradas se clasifican considerando la cercanía del entrevistador: presente, aunque sin interactuar con el entrevistado, y ausente. La primera es una variante de la encuesta presencial, en la que el entrevistador reduce notablemente sus funciones, al limitarse a presentar la investigación y tratar de aumentar la cooperación de los entrevistados. Presentada la investigación, y una vez conseguida la aceptación para colaborar, el entrevistador desaparece y un ordenador portátil interactúa con el entrevistado realizando las preguntas y recogiendo las respuestas. El nivel de interacción entre el entrevistado y el ordenador varía desde aquellos en los que debe pulsar una tecla con la respuesta (videoCASI) hasta los ordenadores que recogen automáticamente la voz del entrevistado, conocidos como audio-CASI o ACASI (Couper et al., 2009; Dillman et al., 2001; Groves et al., 2009; Harmon et al., 2009: 270; Yang et al., 2011).

3. Son datos del total de la investigación, considerados tanto desde el punto de vista cuantitativo $(80 \%)$ como cualitativo (14\%). 
Un segundo tipo lo constituyen las encuestas telefónicas automáticas (telephone audio-CASI o T-audio-CASI), en las que un programa informático lleva a cabo la selección muestral de los números de teléfono y una voz grabada realiza las preguntas y ofrece al entrevistado las posibles respuestas (Couper et al., 2004). La forma de responder será la que permita diferenciar entre diversos tipos de encuesta telefónica automática. El sistema más antiguo se conoce como TDE (touchtone data entry) y se caracteriza porque el entrevistado selecciona la respuesta elegida marcando en el teclado del teléfono el número que acompaña a la opción elegida. Más avances presentan el VR (voice recognition) y el ASR (automatic speech recognition), caracterizados porque el ordenador reconoce la voz del entrevistado cuando elige una respuesta determinada. En todos ellos, las respuestas son automáticamente guardadas en el ordenador, si bien antes de hacerlo repite la opción elegida como modo de verificación (Couper et al., 2009; Groves et al., 2009; Yang et al., 2011).

Este entrevistador virtual desaparece completamente en la encuesta autoadministrada clásica, cuya utilización ha aumentado notablemente en los últimos años con la aparición de las encuestas por correo electrónico y, más recientemente, las encuestas en línea o vía web (Dillman, 2000; Dillman et al., 2009). La penetración y difusión de Internet ha sido una condición necesaria para la utilización de la red como método de recogida de datos, por lo que otras encuestas electrónicas, como la encuesta electrónica autorrellenada (entre otros Saltzman, 1993; Jacobs et al., 1994; SKIM, 1996) y la encuesta electrónica por correo electrónico (Metha y Sivadas, 1995; Tse, 1995 y 1998; Couper et al., 1999) son consideradas los antecedentes inmediatos a las encuestas por Internet (Couper et al., 1999; Fricker y Schonlau, 2002). Ambas demostraron la idoneidad de la utilización del ordenador (del entrevistado) para la recogida de información, al mismo tiempo que constataron que combinan las ventajas del cuestionario autoadministrado tradicional — como la baja deseabilidad social en las respuestas (Groves et al., 2009) — con las ventajas propias del sistema CAPI: utilización de cuestiones complejas, aleatorización de las opciones de respuesta para eliminar el efecto del orden, posibilidad de reducir la influencia de unas preguntas sobre otras, grabación de respuestas cuando se responde el cuestionario que permite el empleo de controles que comprueban la consistencia de las respuestas, etc. Todas estas innovaciones se han trasladado a las encuestas por Internet, tanto a las Computer Assisted Web Inteviewing (CAWI), como a las más populares web site surveys (Kent y Lee, 1999), en la que un determinado sitio web invita a la persona que visita esa página a responder un cuestionario de fácil acceso.

De hecho, uno de los mayores expertos mundiales en el tema señala que, a pesar de su corta historia, se trata de una modalidad que ha tenido un gran impacto $^{4}$ en la investigación con encuestas (Couper y Miller, 2009). Esta gran

4. Otros expertos, por ejemplo Taylor (2000), comparan el desarrollo de la encuesta por Internet al revolucionario descubrimiento de Gallup cuando demostró, en 1936, la utilización de la encuesta para el conocimiento científico de la sociedad. 
utilización está originada por las características intrínsecas de las encuestas web, que serán expuestas en el próximo apartado.

\section{Ventajas e inconvenientes de las diferentes modalidades de encuesta}

La exposición de las ventajas y de los inconvenientes será dividida en tres partes, considerando la teoría del error total propuesta inicialmente por Kish (1965), continuada por Groves (1989) y —-más recientemente- por Weisberg (2005). La revisión llevada a cabo por De Leeuw (2008) tiene en cuenta tres aspectos para detallar las ventajas y los inconvenientes de cada modo de recogida de información. El primero considera la exhaustividad a la hora de abordar todo el universo (errores de no observación), el segundo está referido a la calidad de la información —errores de observación-y, por último, el tercero se refiere a aspectos diversos como el coste, el tiempo necesario para recoger la información, la necesidad de contar con un equipo organizado, etc. Analizaremos cada uno en profundidad en los siguientes apartados.

\subsection{Errores de no observación: ausencia de exhaustividad (del universo objeto de estudio)}

Este criterio tiene en cuenta la posibilidad de contactar con toda la población objeto de estudio, considerando la capacidad que tiene cada modalidad para asegurar una equiprobabilidad de acceso a todo el universo. Dicho de otro modo, se trata de evaluar la adecuada representatividad lograda por cada encuesta, representatividad que implica una elevada cobertura, una alta tasa de respuesta y un error de muestreo conocido y definido.

Comenzando por las ventajas, tradicionalmente la encuesta presencial ha destacado por su gran superioridad en relación con el acceso a toda la población por su elevada cobertura y la alta colaboración de los entrevistados, así como por tener un error de muestreo preciso y conocido (Groves et al., 2009). El segundo tipo de encuesta administrada, la telefónica, presenta como ventajas su bajo coste, facilidad para realizar varios intentos para contactar con un hogar - con lo cual se reduce el número de hogares no contactados - y la posibilidad de llevar a cabo muestreos estratificados, más precisos que los muestreos por conglomerados utilizados habitualmente en la encuesta presencial.

La encuesta por Internet, una derivación de las tradicionales encuestas por correo, presenta como ventaja la posibilidad de acceder a un amplio número de personas con bajo coste y gran rapidez, aspectos difíciles de conseguir en las encuestas administradas (Ilieva et al., 2002). Investigaciones realizadas por Pratesi et al. (2004) con muestras nacionales señalan que la mitad de los cuestionarios se recuperan pasados cuatro días, mucho más rápido que las tradicionales encuestas por correo. Son estimaciones similares a las realizadas por Llauradó (2006) y Valls (2007) en nuestro país. Además, las encuestas por Internet superan las barreras físicas de acceso al entrevistado, tanto en estratos altos (porteros que regulan la entrada, perros guardianes, etc.), como en estratos bajos (peli- 
grosidad de barrios marginales, etc.), al mismo tiempo que permiten acceder a personas muy ocupadas y/o difíciles de localizar con encuestas presenciales y telefónicas.

Algunas de estas potencialidades se ven ligeramente empañadas cuando se consideran las desventajas de cada modalidad. Comenzando con la encuesta presencial, la dificultad (y/o negativa) de los encuestadores de acceder a determinadas zonas, unido al elevado número de hogares vacíos durante gran parte del día (Tucker y Lepkowski, 2008) ha llevado a poner en duda la premisa sobre el elevado porcentaje de población efectivamente cubierta. Además, desde las dos últimas décadas del siglo XX, se han producido importantes descensos en la tasa de respuesta (entre otros, Goyder y Leiper, 1985; Goyder, 1987; Groves y Couper, 1998; Singer, 2006; Stoop, 2006; Stoop et al., 2010; Dillman et al., 2009; Groves et al., 2009; Durrant et al., 2010) que han generado un notable aumento del coste de la investigación, consecuencia del gran número de intentos que debe hacer el encuestador para contactar con una vivienda (Díaz de Rada y Núñez Villuendas, 2008).

La encuesta telefónica presenta como inconveniente la menor capacidad de contactar con todo el universo objeto de estudio, debido a su cobertura más baja, no tanto referida al número de población sin teléfono (en torno a un 0,7\% en España, según el Instituto Nacional de Estadística-INE, 2009), sino más bien por la dificultad de acceder a números telefónicos de hogares (Kalsbeek y Agans, 2008). El empleo de nuevas barreras generadas por los usuarios para filtrar llamadas ${ }^{5}$, el gran abuso en la utilización del teléfono por parte de las empresas de venta y telemarketing, así como la progresiva sustitución de la telefonía móvil por la fija (Peleteiro y Gabardo, 2006; Pasadas del Amo et al., 2011) son aspectos que están reduciendo notablemente su cobertura (Tucker y Lepkowski, 2008). Además, la encuesta telefónica presenta una tasa de respuesta menor que la presencial, fundamentalmente por la facilidad de rechazar la colaboración, si bien la posibilidad de rellamadas sin coste permite rebajar el número de ausencias en el hogar. A estos problemas, debe añadirse la baja tasa de respuesta de determinados colectivos y la sobrerrepresentación de determinados estratos con estudios elevados, jóvenes y clases medias-altas y altas (Wert, 2000).

Las mayores desventajas de las encuestas autoadministradas mediante Internet están relacionadas con la dificultad para localizar muestras representativas (Bradley, 1999; Fox et al., 2003; Diment y Garret-Jones, 2007; Blasius y Brandt, 2010; Baker et al., 2010; De Pedraza, 2010; entre otros). En primer lugar, por problemas de cobertura provocados por el hecho que no todo el mundo tiene acceso a la red. A finales del año 2010 (octubre-noviembre), se

5. Nos referimos, concretamente, al uso de contestadores automáticos y a las pantallas identificadoras del número entrante. Como ejemplo, aludimos a una encuesta telefónica realizada en Navarra en la primavera del año 2009, donde el promedio de teléfonos sin respuesta era del $25 \%$. Esta tasa se incrementa hasta el $60 \%$ cuando en la pantalla del receptor no aparece el número del teléfono que realiza la llamada («No disponible»o «Anónimo»). 
estima que existen en nuestro país 21,6 millones de usuarios de Internet ${ }^{6}$-lo que supone un $54,8 \%$ de la población española (AIMC, 2010) —, si bien otras fuentes (Observatorio Nacional de las Telecomunicaciones y la Sociedad de la Información, 2010) aumentan la cifra a 26,9 millones en el primer trimestre de 2009 - con un 54\% de hogares conectados- e incluso hasta 29.093.984 usuarios, un $62,6 \%$ de la población, según Internet World Stats (2009) 7 . En el verano del año 2010, la revista Investiga señala que «España ocupa el $14^{\circ}$ lugar en el ranking mundial en el número de usuarios de Internet, y sexto en Europa» (Investiga, 2010).

Frente a la cobertura limitada que reflejan estos datos, una gran parte de las investigaciones realizadas a través de Internet destacan la gran rapidez con la que está creciendo el acceso de la población a la red, lo que indica que, dentro de poco tiempo, todo el mundo podrá acceder a ella (Álvarez et al., 2005; Investiga, 2009; Heerwegh, 2009). Algunos de estos estudios ponen el ejemplo de la encuesta telefónica, que, cuando comenzó a utilizarse, había muy poca población con teléfono (Manfreda y Vehovar, 2008) y que, unos años más tarde, ha sido la modalidad más utilizada para la recogida de información (Kalton, 2000; Tourangeau, 2004). De hecho, investigaciones recientes han señalado que, entre 2008 y 2010, ha aumentado notablemente el nivel de digitalización de las personas entre 45 y 64 años (Fundación Telefónica, 2011). Sin embargo, el autor de este trabajo no cree que el crecimiento de la red solucione los problemas de cobertura, entre otras razones porque este crecimiento —al menos en nuestro país — se está ralentizando ${ }^{8}$ y, sin ninguna duda, hay segmentos que nunca accederán a Internet. El Observatorio Nacional de las Telecomunicaciones y la Sociedad de la Información (2010) da cuenta de una relación directa entre la variable estudios terminados y el porcentaje de usuarios de Internet: entre la población con estudios primarios (terminados), el 21\% utiliza Internet, cifra que aumenta al 60,8\% y al $84 \%$ entre los que tienen educación secundaria (primera y segunda etapa, respectivamente), al $89 \%$ entre los que han terminado la FP superior y al 94\% entre los que han terminado los estudios universitarios.

No olvidemos que la red precisa, además de la posesión de un equipamiento (ordenador), una serie de conocimientos para su manejo (Gibson, 2010), algo de lo que carecen importantes sectores de la población (que, por otro lado, disponen y utilizan habitualmente el teléfono móvil, pese a haber sido una tecnología más reciente). Investigaciones realizadas en países donde la expansión de Internet ha aumentado recientemente señalan que el crecimiento de la red

6. Considerando los que han accedido a Internet en el último mes (AIMC, 2010). La mayor parte $(88,2 \%)$ ha accedido desde su hogar; un $23,7 \%$, desde el trabajo; un 5,3\%, desde el centro de estudios, y un 11,2\%, desde otros lugares.

7. En el verano del año 2010, la revista Investiga señala que «España ocupa el $14^{\circ}$ lugar en el ranking mundial en el número de usuarios de Internet, y sexto en Europa».

8. El informe La sociedad de la información en España 2010 señala que «en el año 2010 no se produce apenas variaciones en relación al grado de digitalización con respecto a los datos de 2009» (Fundación Telefónica, 2011). 
está acompañado de un incremento de las diferencias entre la población cubierta y no cubierta (Fricker y Schonlau, 2002; Sparrow y Curtice, 2004; Couper y Miller, 2009; Dillman et al., 2009). Todo esto genera una gran dificultad a la hora de representar a determinados colectivos (Kent y Lee, 1999; Blasius y Drandt, 2010). Tomando, por ejemplo, la situación en Estados Unidos, la tasa de población sin Internet es del 29\%, que aumenta hasta el 50\% cuando se considera a la población que no dispone de conexiones de alta velocidad (Dillman et al., 2009). A juicio de Dillman et al. (2009), las minorías étnicas están totalmente excluidas de las investigaciones mediante Internet, mientras que los votantes y las personas con ideología de izquierdas están sobrerrepresentados, lo que supone serias dudas para la generalización de los resultados.

Un segundo problema relacionado con la representatividad es la gran dificultad para hacer muestreos probabilísticos, que lleva a la utilización de muestras de voluntarios que distan mucho de ser representativas, de ser similares a la totalidad del universo objeto de estudio (Bradley, 1999; Couper y Miller, 2009; Dillman et al., 2009; Baker et al., 2010). Pese a que existen diversos métodos para la elaboración de muestras probabilísticas (Couper, 2000; Manfreda y Vehovar, 2008; Dillman et al., 2009), el incremento en el coste económico que supone su utilización genera que, hoy en día, la mayor parte de las encuestas realizadas por Internet se basen en muestras de voluntarios, en personas autoseleccionadas. La realización de una muestra probabilística precisa de la elaboración de un marco muestral, la selección aleatoria de participantes, las invitaciones para colaborar, el seguimiento de los invitados, los envíos sucesivos para recordar el envío del cuestionario, etc. (Pratesi et al., 2004). Todo ello supone un importante coste económico y, además, retrasa enormemente el comienzo de la investigación (Fricker y Schonlau, 2002).

Algunos expertos consideran que la representatividad podría aumentar empleando paneles que lleven a cabo una adecuada gestión de los panelistas (Duffy et al., 2005; Llauradó, 2006). Se trata de una solución que presenta el problema de todos los muestreos basados en paneles, referidos principalmente a las particularidades y a las diferencias que presentan los que colaboran en paneles respecto a la población general ${ }^{9}$ (Sparrow y Curtice, 2004; Duffy et al., 2005; Schonlau et al., 2010; Baker et al., 2010; Adams, 2011), así como la baja tasa de respuesta (Rao et al., 2010; Zorrilla ${ }^{10}, 2009$; Baker et al., 2010). A esto hay que añadir el cansancio del panelista (Zorrilla, 2009) y la (posible) pérdida de sinceridad al saberse observado. Un trabajo realizado en nuestro país

9. De hecho, en ocasiones puede tratarse de un tipo concreto de autoselección, problema del que dimos cuenta en el párrafo anterior.

A los interesados en profundizar en el tema, recomendamos el reciente trabajo de Baker et al. (2010), donde se encuentran los tipos de paneles en línea, los errores de observación y no observación que presentan, los ajustes para reducir el error y el sesgo, así como una serie de recomendaciones a la hora de trabajar con paneles en línea.

10. Consideramos muy ilustrativo el título del trabajo de este autor: «Nuestras tasas de respuesta son del 40\%, mientras que el sector se sitúa en un 5\%». Baker et al. (2010) señalan que las tasas de respuesta en paneles son inferiores al $10 \%$. 
(Díaz de Rada, 2011) con los datos de una encuesta realizada por el Centro de Investigaciones Sociológicas-CIS (2007a) desvela grandes diferencias entre las encuestas administradas (presenciales y telefónicas) y autoadministradas a través de Internet a un panel ${ }^{11}$, diferencias originadas principalmente por las particularidades de las personas que componen el panel, en línea con hallazgos detectados en otros contextos (Bethlenhem y Stoop, 2003; Chang y Krosnick, 2009; Davidov y Depner, 2009; Dillman et al., 2009).

Otra desventaja que presentan las investigaciones basadas en paneles es la disminución en la sinceridad en las respuestas, debido a la asiduidad de determinadas personas a responder encuestas frecuentemente a cambio de una compensación (Dillman et al., 2009; Baker et al., 2010; Adams, 2011). En la investigación realizada en nuestro país con datos del CIS, un $75 \%$ de los entrevistados a través de Internet (panel) habían sido encuestados antes, frente al 60\% de los entrevistados por un encuestador (Díaz de Rada, 2011). De hecho, en uno de los últimos trabajos publicados, se señala que los desafíos de las encuestas por Internet son la construcción de muestras probabilísticas y el análisis crítico de la viabilidad en la utilización de paneles (Couper y Miller, 2009; Baker el al., 2010). Muy relacionado con esto, aparece otra crítica que tiene que ver con la dificultad de hacer llegar el cuestionario a un individuo concreto, lo que dificulta el cumplimiento de diversas cuotas que representen adecuadamente a todos los estratos de la población objeto de estudio.

El tercero de los problemas referidos a la representatividad en las encuestas administradas mediante Internet está relacionado con la menor tasa de respuesta de las encuestas por Internet (entre otros, Fricker y Schonlau, 2002; Heerwegh y Loosveldt, 2009), en torno un 11\% inferior según Manfreda et al. (2008), aunque algunas escasas investigaciones han obtenido tasas de respuestas similares a otras modalidades de recogida de información (Ilieva et al., 2002; Heerwegh, 2009). En el estudio realizado del CIS citado anteriormente (Díaz de Rada, 2011), fueron seleccionados 35.000 panelistas $^{12}$, de los que se obtuvieron 530 respuestas, lo que da una idea de la escasa tasa de cooperación.

Una estrategia tradicionalmente empleada por las encuestas administradas para aumentar la cooperación ha sido el empleo de incentivos (Dillman et al., 2009), si bien éstos consiguen una menor eficacia en las encuestas a través de la web (por ejemplo: Fricker et al., 2005; Lamas, 2007; Manfreda y Vehovar, 2008). Numerosas investigaciones (entre otras, Görtiz, 2006; Van den Brakel et al., 2006; Bonke y Fallesen, 2010; Preece et al., 2010; Rao et al., 2010)

11. Las características técnicas de la investigación pueden consultarse en Centro de Investigaciones Sociológicas, 2007b.

12. Consideramos que es interesante reproducir aquí el texto exacto extraído de la metodología del estudio 2676 del CIS: «Previamente a la puesta en funcionamiento del cuestionario web, ODEC compró a uno de sus proveedores (SSI Sampling) una base de datos de 35.000 panelistas acotados de la misma manera que en las cuotas establecidas, a los que se les "invitó" a participar en este estudio mediante un link con el que podían acceder sólo a través de su correo electrónico, lo que garantiza que en el test hayan participado sólo los "invitados"” (CIS, 2007b). 
destacan que el uso de incentivos materiales (regalos) incrementa la tasa de respuesta, lo que demuestra también la elevada influencia de las gratificaciones en el mantenimiento de los panelistas (Görtiz, 2008). La mayor parte de investigaciones sobre el tema constatan la mayor influencia de las gratificaciones en metálico frente a los regalos, así como los envíos antes de responder el cuestionario en vez de la "promesa» de gratificación cuando se responda (Birnholtz et al., 2004; Dillman et al., 2009).

\subsection{Errores de observación: calidad de la información recogida}

Este apartado considera la calidad de la información recogida por cada modalidad, y en él se aborda el error del entrevistador, del entrevistado, del instrumento de medida y de las modalidades de recogida de información.

Siguiendo con el mismo orden expuesto en las páginas anteriores, la encuesta presencial destaca por la reducción del número de respuestas evasivas, el conocimiento de la identidad de la persona entrevistada, la posibilidad de limitar la influencia de otras personas en las respuestas, la fácil utilización de preguntas filtro, el uso de preguntas complejas ${ }^{13}$, la posibilidad de mostrar objetos al entrevistado, así como la facilidad para obtener datos secundarios sobre el entorno del entrevistado (información que, en ocasiones, puede estar muy influida por la propia subjetividad del entrevistador). La posibilidad de eliminar la contaminación entre preguntas ${ }^{14}$, así como la reducción de los efectos de primera y última opción cuando la entrevista se lleva a cabo con el apoyo de un ordenador (Computer Assisted Personal Interviewing-CAPI), genera una alta calidad en la contestación del cuestionario. Además, se cuenta con una persona que responda a las dudas originadas en la lectura del cuestionario, si bien esta última es una «ventaja relativa», puesto que los entrevistadores pueden dar un sesgo a cada pregunta, lo cual aumenta la influencia de cada entrevistador en la respuesta.

En la enumeración de las ventajas de la encuesta telefónica, aparece la menor influencia del entrevistador, la facilidad para controlar al entrevistador y detectar posibles errores en la realización de las entrevistas (Groves y Kahn, 1979; Fowler y Manglione, 1980; Presser et al., 2004) y una mayor sensación de anonimato. Mayor sensación de anonimato que la presencial, pero menor que la autorrellenada. Esta sensación de anonimato no implica necesariamente una mayor adecuación para el estudio de temáticas sensibles, porque la literatura presenta ejemplos de investigaciones donde la encuesta presencial muestra una menor deseabilidad social (entre otros, Groves y Kahn, 1979; De Leeuw y Van der Zouwen, 1988; Körmendi, 1988; Aquilino, 1994; Groves et al., 2009; Holbrook et al., 2003), mientras que otras demuestran que la encuesta telefónica es más adecuada para el estudio de este tipo de temáticas (Sykes y

13. Puesto que la entrevista se realiza con un entrevistador y éste ha recibido formación sobre cómo llevarla a cabo.

14. Término que se refiere a la influencia que unas preguntas ejercen sobre otras. 
Collins, 1988). La encuesta asistida por ordenador, al integrar distintos procesos de la investigación (como el muestreo, la aplicación del cuestionario y la tabulación mientras se recoge la información), permite detectar cualquier incidencia metodológica en el momento en el que se produce, de modo que sus consecuencias dañinas (en tiempo y dinero) disminuyen considerablemente (Wert, 2000).

La encuesta por Internet, por su parte, presenta grandes ventajas consecuencia de las mejoras en el instrumento de recogida de información, al pasar de un cuestionario de papel a un innovador documento audiovisual que interactúa con el entrevistado empleando recursos visuales y auditivos (Couper, 2000; Fox et al., 2003; Manzano et al., 2003; Álvarez et al., 2005; Thomas y Klein, 2006; Tourangeau et al., 2007; Steeh y Piekarski, 2008; Delavande y Rohwedder, 2009; Couper, 2008). Las encuestas web presentan una interacción más visual con el encuestado, al permitir mostrar fotografías, colores, logotipos ${ }^{15}$, etc. (Manzano y Andreu, 2000), para que el entrevistado los analice o exprese su opinión sobre ellos, lo que las configura como una modalidad flexible e interactiva, fundamentalmente cuando se compara con la encuesta telefónica (Fricker, et al., 2005; Chang y Krosnick, 2010). Estos factores reducen el número de preguntas sin responder (Heerwegh, 2009; Bech y Bo Kristensen, 2009), proporcionan respuestas más largas en las preguntas abiertas (Fricker y Schonlau, 2002), al mismo tiempo que permiten la utilización de reglas de consistencia cuando se responde el cuestionario, lo que supone una mayor veracidad de las respuestas obtenidas.

La encuesta autocumplimentada ofrece al entrevistado la posibilidad de reflexionar, de contestar a su ritmo, seleccionando su mejor momento y empleando el tiempo que precise, lo que facilita la consulta de documentos, archivos, etc. Al no existir encuestador, no se producen sesgos por su influencia, lo que implica una mayor garantía de anonimato que ayuda a que los entrevistados expresen unas respuestas más críticas, menos aceptadas socialmente (Kreuter et al., 2008), lo que podría explicar la menor aquiescencia de estas encuestas (Heerwegh, 2009). Esta menor deseabilidad social (McCullough, 1998; Dillman, 2000; Heerwegh, 2009) genera estimaciones más precisas en encuestas políticas y en temáticas delicadas (Duffy et al., 2005). Frente a la encuesta por correo, en la que el entrevistado puede analizar el cuestionario previamente y comenzar por donde desee, el cuestionario administrado por Internet impone un flujo al entrevistado, una secuencia de obligado cumplimiento (Fox et al., 2003). Además, el cuestionario proporciona información sobre como ha sido respondido: navegador utilizado, tiempo empleado en su respuesta, interrupciones e inicios posteriores, así como la modificación de respuestas dadas (Domínguez Álvarez, 2008).

Los aspectos expuestos en los párrafos anteriores deben considerarse teniendo en cuenta las desventajas de cada modalidad. Las principales críticas de la encuesta presencial están relacionadas con la influencia del entrevistador, principalmen-

15. Con gran calidad, al ser presentados en la pantalla del ordenador. 
te en temáticas sensibles (entre otros, Catania et al., 1996; Holbrook et al., 2007; Tourangeau y Smith, 1996; Verge Mestre, 2009). Otras desventajas aluden a la lentitud para la recogida de información (en relación con otras modalidades) y el elevado coste económico derivado de la necesidad de contar con un grupo de encuestadores y supervisores debidamente formado. La necesidad de controlar a los entrevistadores, así como la dificultad de realizar segundas visitas en las viviendas con personas ausentes en el momento de llevar a cabo la entrevista, son otras desventajas no menos desdeñables.

Las principales críticas de la encuesta telefónica aluden a la forma en la que se produce la comunicación, lo que recomienda no realizar preguntas largas o con un elevado número de respuestas, así como el hecho que no sea posible mostrar objetos al entrevistado ni obtener información de su entorno. Además, los cuestionarios se responden peor que los realizados por encuesta presencial, como ha señalado la teoría de la complacencia (survey satisfacing), cuando demuestra que los entrevistados por teléfono presentan menos cooperación y compromiso con la entrevista, lo que genera un mayor número de respuestas deseables, mayores tasas de aquiescencia y menor diferenciación en las escalas (Krosnick, 1991 y 1999; Nicolaas, 2000).

Unos párrafos más atrás, dimos cuenta de las ventajas que supone —en la encuesta por Internet - la utilización de recursos audiovisuales en el diseño del cuestionario, si bien es importante tener en cuenta también que la pantalla del ordenador suele leerse con más rapidez e impaciencia que los textos en papel, lo que lleva a que determinados entrevistados lleguen a escanear el texto en vez de leerlo en detalle (Graft, 2002; Heerwegh y Loosveldt, 2009b). Por este motivo, es esencial poner una gran atención al diseño del cuestionario empleando preguntas y respuestas cortas, concisas y presentadas con suma claridad (Graft, 2002). Otro aspecto negativo, señalado por varias investigaciones, es el incremento de tareas que debe asumir el entrevistado por la ausencia o eliminación del encuestador, incremento de tareas que - en ocasiones - genera descensos en la calidad de la respuesta (Japec, 2006).

\subsection{Aspectos complementarios}

El tercer criterio recoge aspectos variados relacionados con la duración del cuestionario, el coste económico, el tiempo necesario para recoger la información, la necesidad de contar con un equipo organizado, la posibilidad de corregir problemas ocurridos en la administración del cuestionario, etc.; circunstancias que quedan fuera de los errores de no observación y observación.

Procediendo con las ventajas, la encuesta presencial destaca por la posibilidad de realizar encuestas más largas, una de las ventajas exclusivas de esta modalidad. La encuesta telefónica cuenta como aspectos positivos su bajo coste (en torno a un tercio del coste de la encuesta presencial), la necesidad de contar con menos personal para su administración y la gran rapidez en la disposición de resultados, lo que la hace adecuada para la realización de estudios tácticos que buscan medir en caliente determinadas situaciones (Wert, 2000). 
La ventaja principal de la encuesta por Internet es la exigencia mínima de personal y de recursos para realizar el trabajo de campo, además de ser un sistema económico de recogida de información (Álvarez et al., 2005). Se produce una importante disminución de costes, al ser eliminados los gastos de administración e introducción de la información, así como la impresión y el fotocopiado de cuestionarios y el franqueo para el envío (Fox et al., 2003); si bien aparece una nueva partida de gastos relacionada - en encuestas a paneles- con el cuidado del panel, porque es necesario dedicar unos importantes recursos económicos al cuidado del panelista y a la puesta al día de la base de datos (Duffy et al., 2005; Hoogendoor y Daalmans, 2009; Toepoel et al., 2009). Es preciso aludir también a la comodidad en la aplicación y el reducido número de errores de trascripción al volcarse la información automáticamente (Kent y Lee, 1999; Ilieva et al., 2002; Álvarez et al., 2005; Manfreda y Vehovar, 2008; Vehovar et al., 2008). La encuesta por Internet produce también un mayor control sobre el encuestado, en la medida que se conoce el tiempo transcurrido entre el visionado de la entrevista y el proceso de respuesta (Domínguez Álvarez, 2008), al tiempo que —al tener como remitente el IP del equipo donde se ha respondido- limita las posibilidades de responder varios cuestionarios (en una misma investigación).

Como aspectos negativos, la encuesta presencial necesita contar con un gran equipo de encuestadores y supervisores formados, tiene un alto coste económico y la recogida de información precisa de bastante tiempo (entre otros, Revilla, 2010), si bien éste disminuye notablemente cuando se utilizan encuestas presenciales asistidas con ordenador. La encuesta telefónica presenta como limitación la necesidad de contar con encuestas cortas, por la facilidad para poner fin a la entrevista.

En cuanto a los aspectos negativos de la encuesta por Internet, debe considerarse la desconfianza en la seguridad de la red, aspecto vital para determinados segmentos no familiarizados con ésta (Manfreda y Vehovar, 2008). Al igual que en las tradicionales encuestas postales, se produce incertidumbre sobre la identidad de quien responde el cuestionario y la posible influencia de otras personas en su cumplimentación.

Sintetizando, las ventajas más importantes de la encuesta por Internet son su gran rapidez, que permite una reducción importante del trabajo de campo; la mejora en la respuesta, por la posibilidad de introducir elementos audiovisuales en el cuestionario que facilitan su lectura y respuesta, y el menor coste de la investigación, debido a la eliminación de los encuestadores (cuando se compara con encuestas presenciales y telefónicas). Entre sus grandes inconvenientes, se encuentran los errores de cobertura, la escasa aleatoriedad de las muestras y la menor tasa de respuesta.

\section{Comparativa entre modalidades de recogida de información}

Las desventajas de las encuestas web señaladas en el apartado anterior quedan debilitadas cuando se constata que algunas — más que referidas a las encuestas 
web- están relacionadas con la metodología de la encuesta como tal. ¿Qué modalidad presenta cobertura total?; ¿hay alguna que garantice una tasa de respuesta total?, ¿y ausencia de errores de medición?... De hecho, la enumeración de desventajas queda mitigada cuando se consideran las distintas investigaciones que comparan los resultados de encuestas web con encuestas presenciales, telefónicas y por correo.

Comenzaremos por estas últimas, al haber sido objeto de más investigaciones. Comparadas con las encuestas postales tradicionales y las encuestas por fax, Cobanoglu et al. (2001) dan cuenta de la gran rapidez en la recepción de los cuestionarios respondidos: una media de cinco a seis días, frente a los cuatro días del fax y los dieciséis de la encuesta por correo. La diferencia con el fax se explica aludiendo a que, en el año 2000, las personas consultaban con más frecuencia el fax que el correo electrónico ${ }^{16}$, hecho que ha cambiado totalmente en la actualidad. En esta investigación, se señala que el número de preguntas no respondidas en la encuesta web es similar a la conseguida en la encuesta por correo tradicional $(20 \%)$ e inferior a la encuesta por fax (25\%), si bien la mayor parte de investigaciones destacan que la encuesta por Internet consigue una mayor calidad en la respuesta y unas respuestas más largas a las preguntas abiertas (Fricker y Schonlau, 2002; Kwak y Radler, 2002; Bech y Kristensen, 2009).

Una de las últimas investigaciones realizadas en nuestro país, concretamente el estudio sobre "la situación de los andaluces en el extranjero" (Instituto de Estudios Avanzados del Consejo Superior de Investigaciones CientíficasIESA) ${ }^{17}$, modifica los hallazgos realizados por Cobanoglu et al. (2001) respecto a la rapidez de respuesta. En el primer mes, la encuesta web recoge un 26,5\% de los cuestionarios, frente al $18 \%$ de la encuesta postal, pero en el segundo mes la tendencia se invierte y la encuesta por correo proporciona un $48 \%$ de los cuestionarios, frente al $29 \%$ de la encuesta web $^{18}$. Dicho de otro modo, dos meses después del inicio de la investigación, la encuesta por correo ha sido respondida por un $66 \%$ de los entrevistados y la encuesta web, por un 55\%. En cuanto al número de respuestas proporcionado por cada modalidad, otro de los aspectos señalados en el párrafo anterior, los que responden a través de Internet proporcionan un mayor número de respuestas que los que optan por el cuestionario por correo tradicional: el $18 \%$ de los que responden al cuestionario por Internet responden a todas las preguntas y el 54\% dejan sin

16. Las personas seleccionadas recibieron un correo electrónico donde se les informaba que eran invitadas a participar en una encuesta que estaba colgada en un servidor web.

17. Realizado por el Instituto de Estudios Avanzados del Consejo Superior de Investigaciones Científicas (IESA-CSIC) en el año 2010 y patrocinado por la Consejería de Gobernación de la Junta de Andalucía. En el anexo, se muestran las características técnicas de la investigación.

18. Es preciso indicar que el propio entrevistado selecciona la forma en la que prefiere responder el cuestionario, como se explica en el anexo. De los 2.446 cuestionarios respondidos, el 82\% fueron respondidos a través del correo tradicional; un 14,7\%, mediante Internet, y un 1,5\%, con una encuesta telefónica. El resto $(1,8 \%)$ se respondieron mediante usos combinados de correo postal e Internet (21 cuestionarios, 0,9\%), correo postal y CATI (19 cuestionarios, $0,8 \%$ ) e Internet y CATI (4 cuestionarios, $0,2 \%$ ). 
responder una pregunta, porcentajes que descienden hasta el $11 \%$ y el $28 \%$ entre los que responden al correo tradicional. Un $16 \%$ de estos últimos deja sin responder seis y más preguntas, porcentaje que desciende al 6,2\% entre los entrevistados a través de Internet ${ }^{19}$.

Otro aspecto relacionado con la calidad de la cumplimentación del cuestionario, las respuestas proporcionadas a las preguntas abiertas, muestra menores diferencias que las detectadas por investigaciones realizadas en otros contex$\operatorname{tos}^{20}$. La encuesta web consigue un mayor número de respuestas en todas ellas $^{21}$, diferencia que es significativa únicamente en una de éstas, la referida a los problemas o a las necesidades principales: el $89 \%$ de los entrevistados a través de Internet señala un problema, frente al $76 \%$ de los que responden por correo, un $61 \%$ dice el segundo problema - por correo el 51\% — y la mitad de los entrevistados declara un tercer problema (por correo un $42 \%)^{22}$. Una forma más detallada de analizar estas preguntas es tener en cuenta la longitud de las respuestas proporcionadas, considerando el número de palabras o caracteres expresados en cada una. Este análisis, pese a ser mucho más detallado, apenas desvela aportaciones relevantes, puesto que únicamente dos variables presentan diferencias significativas: los problemas o las necesidades principales en segundo lugar (pregunta D1) y el motivo más importante por el que sigue en el país ${ }^{23}$ (pregunta F3). La encuesta por Internet siempre es la que proporciona unas respuestas más largas.

Otro aspecto interesante que refleja este estudio son las diferencias expresadas por los entrevistados en el grado de confianza de distintas instituciones relacionadas con la que patrocina el estudio. La encuesta por Internet ofrece

19. En definitiva, los entrevistados mediante encuesta postal dejan una media de 3,26 preguntas sin responder, frente a las 2,34 preguntas que no responden los entrevistados por Internet.

20. El cuestionario - de 59 preguntas y 150 variables - tiene cinco preguntas abiertas donde el entrevistado puede expresarse, esto es, además de otras que se responden con un número (fecha, número de veces, ingresos, etc.). De estas cinco, serán consideradas únicamente cuatro, porque una de ellas (pregunta D4 del cuestionario) es respondida únicamente por los que han acudido a los servicios sociales del país. Se detallan a continuación las preguntas incluidas en nuestros análisis:

D1. En estos momentos, ¿cuáles son, por orden de importancia, sus problemas o necesidades principales? (Por favor, especifique lo mejor posible hasta un máximo de 3 respuestas.)

F3. ¿Cuáles son los motivos que le retienen actualmente en el "pais»? (Dígame hasta 3 por orden de importancia.)

G6. ¿Cuáles fueron los motivos principales por los que emigró de Andalucía y España? (Dígame hasta 3 por orden de importancia.)

G7. Y, en concreto, ¿podría decirme por qué motivos eligió establecerse en ese país? (Dígame hasta 3 por orden de importancia.)

21. Diferencia que es mayor en los problemas importantes en segundo y tercer lugar (pregunta D1, ver nota al pie anterior), así como en el segundo y tercer motivos para establecerse en el país (pregunta G7, ver nota al pie anterior).

22. Esto es, no hay diferencia significativa entre el tipo de cuestionario respondido en las preguntas sobre los motivos que le retienen en el país, los motivos principales por los que emigró y los motivos que le llevaron a instalarse en ese país.

23. En este caso, la diferencia es únicamente por el número de caracteres, y no considerando el número de palabras. 
unas respuestas más negativas; un menor grado de confianza de la Junta de Andalucia, las diputaciones y los ayuntamientos. Esto contrasta con el estudio realizado por el CIS en el año 2007, donde los entrevistados a través de Internet presentan respuestas más positivas, al declarar una mayor confianza en las encuestas, un mayor seguimiento de las noticias sobre éstas y una mayor creencia en su utilidad (Díaz de Rada, 2011). Se trata de un aspecto no muy abordado en la investigación sobre el tema y que necesita de mayor investigación.

En cuanto al número de respuestas obtenidas, y aunque Cobanoglu et al. (2001) encuentran una mayor tasa de respuesta en la encuesta web (un 42\%, frente a la encuesta por correo, un 26\%, y por fax, un 17\%), la mayor parte de la literatura atribuye a la encuesta por Internet una tasa de respuesta inferior a la lograda por la encuesta por correo (Cook et al., 2000; Converse et al., 2008; Manfreda et al., 2008; Shih y Fan, 2008; Couper y Miller, 2009). Otra diferencia muy importante es que la encuesta por Internet no aumenta la tasa de respuesta cuando se realizan envíos sucesivos del cuestionario (Cook et al., 2000; Kwak y Radler, 2002; Porter y Whitcomb, 2006; Shih y Fan, 2008; Kaplowitz et al., 2004), una de las estrategias más efectivas para aumentar la tasa de respuesta en encuestas postales (Heberlein y Baumgarter, 1978 y 1981), pero es más eficaz el empleo de envíos previos donde se presenta la investigación (Heerwegh, 2005; Porter y Whitcomb, 2006; Shih y Fan, 2008; Muñoz Leiva et al., 2009). Ahora bien, es sin duda en el coste donde las encuestas web presentan unas mayores ventajas, con un promedio de 2,5 dólares por cuestionario respondido, tres veces inferior a las encuestas por fax (7 dólares) y cuatro veces menos que las encuestas por correo (10 dólares).

Algunos de estos resultados quedan matizados cuando se considera la población con menor acceso a la web, como demuestra una investigación realizada en Dinamarca a los mayores de 55 años (Bech y Bo Kristensen, 2009). En Dinamarca, el 86\% de la población entre 40 y 59 años es usuaria de la red, porcentaje que se reduce al 57\% entre la población entre 60 y 74 años. La encuesta por correo logra una tasa de respuesta del $41 \%$, que se reduce al $17 \%$ en el caso de la encuesta web; diferencias menores a las localizadas en investigaciones similares ${ }^{24}$. La cooperación por regiones apenas varía, con una importante infrarrepresentación de las personas con edades entre 50 y 54 y 65-75 años. Cooperan menos en las encuestas web aquellos con menores ingresos, menor educación y los que viven solos. Considerando la calidad de la respuesta, la encuesta web presenta una menor tasa de respuesta parcial, diferencia que es interpretada aludiendo a que este tipo de encuesta ayuda a responder, al motivar más a los entrevistados, además de que el propio sistema puede indicar al entrevistado que ha olvidado responder una pregunta ${ }^{25}$ (Bonsjak y Tuten,

24. Dannentun et al. (2007), por ejemplo, consiguen unas diferencias mayores al lograr tasas de cooperación del 55\% y de $15 \%$, respectivamente.

25. Es posible utilizar cuestionarios que no permitan dejar sin responder una pregunta, práctica nada recomendable, porque puede producir frustración en los entrevistados y numerosos abandonos en el proceso de respuesta. 
2003). Aunque la encuesta por web presenta un menor coste por cuestionario respondido (9,87 euros en correo convencional y 6,13 euros en web), la escasa diferencia entre ambas no compensa la baja tasa de respuesta de la encuesta web. Mucho más cuando otros estudios han localizado diferencias mayores. Jones y Pritt (1999) señalan que una encuesta web cuesta la mitad que una encuesta por correo; Akl et al. (2005), un tercio, y McDonald y Adam (2003), un $24 \%$.

Hay un gran número de investigaciones que comparan las encuestas web y las telefónicas, y que señalan grandes diferencias en la tasa de respuesta, la calidad de la respuesta, la no respuesta parcial y la presencia diferente de efectos de respuesta (Bandilla et al., 2003; Roster et al., 2004; Manfreda et al., 2008; Fricker et al., 2005; Christian et al., 2008). A las desigualdades en la selección muestral (mayor número de hombres, jóvenes y altos estudios en las encuestas por Internet), hay que añadir las grandes diferencias en las variables específicas de la encuesta (variables de contenido). Así, Schonlau et al. (2003) encuentran diferencias significativas en 29 de las 37 preguntas de un cuestionario de salud, si bien señalan que son mayores en las preguntas de actitud que en las de comportamiento. De la misma forma, en una investigación sobre actitudes hacia el medio, Bandilla et al. (2003) muestran que en 22 de los 30 ítems del cuestionario (un $73 \%$ ) hay diferencias en sus respuestas. Similares conclusiones señalan Sparrow y Curtice (2004), en su investigación sobre actitudes políticas e intención de voto; Roster et al. (2004), en una investigación sobre valores de la comunidad, y Fricker et al. (2005), en las actitudes hacia la ciencia y la tecnología: los entrevistados a través de Internet tienen un mayor conocimiento tecnológico.

El número de investigaciones que comparan las encuestas web con las presenciales es muy inferior, fundamentalmente por la menor utilización de éstas a favor de las telefónicas (Kalton, 2000; Tourangeau, 2004). Entre todas, destacamos la realizada por Duffy et al. (2005), en la que señala que los que participan en encuestas web son políticamente más activos, adoptan antes la tecnología, están más informados, viajan más y comen fuera de casa con más frecuencia que los que responden encuestas cara a cara. Los encuestados a través de la web son más liberales, expresan más sus opiniones y, comparado con la encuesta presencial, presentan una menor tasa de no respuesta en la declaración del voto (Duffy et al., 2005). En cuanto a las características de la población cubierta, es importante resaltar que este tipo de encuestas logran una mayor participación de personas que no cooperan con otros métodos, personas muy ocupadas, más liberales y con mayor nivel educativo (Duffy et al., 2005).

Investigaciones más recientes, como la realizada por Heerwegh y Loosveldt (2009b), analizan el impacto en la calidad de la respuesta de la encuesta web generada por el diferente tipo de interacción y las nuevas tareas que debe realizar el encuestado en este tipo de encuestas. En la encuesta cara a cara, la comunicación no verbal tiene un gran protagonismo, lo que precisa de un menor esfuerzo cognitivo. La encuesta telefónica, al permitir hacer otras tareas (ver la televisión, escuchar la radio, etc.), puede incidir en pérdidas de calidad en 
la respuesta. Este efecto será mayor en la encuesta por Internet por el mayor esfuerzo cognitivo que se precisa del entrevistado, al tener que leer las preguntas y las respuestas (no sólo escuchar como en la telefónica y presencial), comprender las escalas utilizadas, leer las instrucciones sobre la forma de responder, etc. Es decir, desaparece la ayuda de la comunicación no verbal de la encuesta presencial y los recursos auditivos de la telefónica, lo que precisa de un mayor esfuerzo del entrevistado (Tourangeau et al., 2000; Japec, 2006). El punto de partida es la teoría del survey satisficing (Krosnick, 1991 y 1999) — que tan buenos resultados ha proporcionado en encuestas telefónicas-, que lleva a considerar a los investigadores — como hipótesis - que los entrevistados a través de la web lo sufren más que los del resto de modalidades.

Cuando este planteamiento es aplicado al personal de la universidad católica de Leuven (Bélgica) mediante una encuesta web y otra presencial, se encuentran que la primera se responde más rápido, lo que induce a pensar que se ha respondido sin prestar mucha atención. Además, el número de respuestas «No sabe» es 2,6 veces más alto en la encuesta web que en la presencial, a su juicio porque el entrevistador utiliza técnicas de indagación (probings) para estimular la respuesta. La no respuesta parcial es muy baja en ambas encuestas, si bien es ligeramente superior en la presencial. El análisis de las preguntas basadas en escalas (entre el valor 1 y el 5) desvela una mayor elección de la categoría central en la encuesta por Internet, así como una ligera diferencia en los valores extremos de la escala. Las mayores diferencias se localizan en la tasa de respuesta: un $93 \%$ en la encuesta presencial y un $52,5 \%$ en la telefónica.

Una investigación llevada a cabo en mayo del 2007 entre los alumnos de esta misma universidad trata de comprobar si las encuestas presenciales (con un coste económico muy elevado) consiguen una calidad en la respuesta superior que las realizadas a través de Internet (Heerwegh, 2009). La calidad en la respuesta se mide considerando el número de no respuestas («No sabe» $\mathrm{y}$ «No responde»), la diferenciación en la respuesta y el efecto aquiescencia. En principio, se esperaba que los entrevistados a través de la web expresaran una menor diferenciación en las escalas, como consecuencia - por un lado- del efecto satisficing y - por otro- por el gran número de preguntas de batería incluidas en las encuestas a través de la web. Asimismo, se planteaba como hipótesis que la encuesta por Internet tendría una menor aquiescencia que la presencial, debido a la ausencia de entrevistador en la primera (Heerwegh, 2009).

Los resultados son similares en la tasa de respuesta y con escasas diferencias en la calidad de respuesta. La encuesta por Internet presenta una ligera respuesta menor, que no llega a ser significativa, y se obtienen resultados similares en relación con la aquiescencia y la diferenciación en las escalas. Salvo la no respuesta parcial, apenas hay variación en la calidad de respuesta. Estos hallazgos, diferentes a los mostrados por otras investigaciones, son explicados aludiendo a que pueden estar producidos porque se trata de una encuesta realizada a personas jóvenes y universitarias, muy acostumbradas a la utilización de la red (Heerwegh, 2009). Esta similitud cambia cuando se considera la deseabilidad social, más presente en la encuesta presencial, aunque con menos influencia de la esperada. Estas dife- 
rencias son explicadas aludiendo a la ausencia de encuestador en la encuesta por Internet, lo que implica una mayor distancia social de esta última, que disminuye el número de respuestas deseables socialmente (Heerwegh, 2009).

En España, se han realizado escasas investigaciones sobre el tema y apenas hay publicaciones al respecto, sin duda por la lenta expansión de Internet. En el año 2005 , el 42,1\% de la población española tenía acceso a Internet y ocupaba la posición 19 en la lista de países con mayor difusión de la red; diez puntos por debajo de la UE (Fernández Aedéval, 2006). Pese a esta situación, en este año varias empresas habían desarrollado paneles que representaban adecuadamente a la mayor parte de la población española. Algunos investigadores compararon encuestas telefónicas con encuestas en línea y dieron cuenta de la similitud en la posesión de electrodomésticos y automóviles, así como unas ligeras diferencias en tecnología en el hogar entre los menores de 55 años (López, 2006), lo que les llevó a recomendar la utilización de paneles cuando la población objeto de estudio sea menor de esa edad ${ }^{26}$ (López, 2006). La experiencia de otras investigaciones (Llauradó, 2006) señalan que apenas hay diferencia entre los que participan en paneles y la población general, si bien los jóvenes y los adolescentes tienen más predisposición a participar.

Un estudio realizado por Díaz de Rada (2010) detecta grandes diferencias entre los que responden a un mismo cuestionario mediante entrevista presencial e Internet (panel), tanto en los rasgos sociodemográficos como en las variables propias de la investigación (variables de contenido). Estas diferencias se mantienen cuando se consideran — entre los entrevistados por encuesta presencial- únicamente los que tienen acceso a Internet. Las diferencias en las variables de contenido persisten aún cuando se elimina la influencia de las variables sociodemográficas con más influencia (edad, estudios y relación con la actividad).

En cualquier caso, algunos expertos estimaban que las encuestas a través de la web habían superado el 10\% de los estudios contratados en España (López, 2008). Ahora bien, es posible que se trate de una estimación a la baja, ya que la investigación sobre la industria española de investigación de mercados y opinión pública (realizada por AEDEMO y ANEIMO) destaca que el 14\% de las encuestas realizadas en el año 2007 en España han sido a través de Internet, con un crecimiento del $24 \%$ respecto al año anterior (Rodríguez Esteban, 2008). En el año 2008, la investigación en línea representa un 3,6\% del total del mercado, según AEDEMO, con una estimación del 6\% para el año 2010 (Zorrilla, 2010), aún muy lejos del $20 \%$ que supone esta investigación en Europa. En 2009, prácticamente una de cada cuatro encuestas (exactamente un 24,2\%) realizadas en España ha sido a través de Internet (ANEIMO, 2010).

A mediados del año 2010, la publicación del estudio sobre el sector de la investigación de mercado referente a 2009 da cuenta de la tendencia hacia la

26. Hay un menor acceso por parte de los mayores de 55 años, clase modesta o determinadas temáticas, características que comparten también las personas que carecen de teléfono en su hogar (y que se quedan sin cubrir en las encuestas telefónicas). 
recogida de datos de forma electrónica, automática y en línea, que supone un $24,2 \%$ de la información recogida (ANEIMO, 2010). Comparando la situación con el año anterior, señalan que la recogida automatizada ha aumentado un 16,2\%, mientras que la llevada a cabo a través del teléfono o de forma presencial ha disminuido en un $16,6 \%$ y en un $15,4 \%$, respectivamente. Estimaciones realizadas en otros contextos señalan que alrededor del $85 \%$ de la investigación actual se está llevando a cabo reemplazando las encuestas presenciales y telefónicas (Baker et al., 2010).

\section{Conclusiones}

Este trabajo ha comenzado describiendo el gran desarrollo de las modalidades de recogida de información experimentado en las últimas dos décadas del siglo XX, debido, fundamentalmente, a los cambios en la sociedad y al encarecimiento de los procesos de recogida de información. Dentro de los primeros, debemos considerar el aumento del número de personas que trabajan fuera de casa, el alargamiento de las jornadas laborales, el incremento en el número de hogares unipersonales y el progresivo descenso del tamaño de los hogares; elementos que reducen el tiempo de estancia en la propia vivienda.

La dificultad de la investigación con encuesta para encontrar a personas en su domicilio a las que entrevistar supuso el desarrollo de la encuesta telefónica, que permite ajustar las horas para entrevistar en los momentos en los que hay más gente en casa (puesto que no se producen desplazamientos de los entrevisadores), y el renacer de la encuesta por correo en el último cuarto del siglo XX. En la primera década del siglo XXI, el predominio de la encuesta telefónica está siendo amenazado por el gran desarrollo del telemarketing y la venta a través del teléfono, el incremento del número de barreras empleadas por los usuarios para evitar responder al teléfono, así como el descenso de cobertura del teléfono fijo y el aumento del móvil (Tucker y Lepkowski, 2008). Estos factores han supuesto importantes aumentos económicos y un alargamiento de los trabajos de campo, que, unidos al cambio en las actitudes de la población hacia la privacidad y la confidencialidad (Singer y Presser, 2008; Singer, 2004; Singer et al., 2003), han generado el desarrollo de nuevas herramientas para la realización de encuestas (Fricker et al., 2005).

Una de éstas ha sido la encuesta por Internet, «heredera» de la encuesta electrónica autorrellenada y la encuesta electrónica por correo electrónico. A las ventajas de las encuestas tradicionales por correo, las realizadas mediante Internet añaden una mayor rapidez en la recogida de información, una mejora en el diseño del cuestionario derivados de la utilización de elementos audiovisuales y el menor coste, al prescindir de entrevistadores y franqueo para el envío de los cuestionarios. Las desventajas están relacionadas con la baja cobertura, los problemas en la elaboración de un muestreo aleatorio y la menor tasa de respuesta. El objetivo de este trabajo era analizar hasta qué punto los resultados obtenidos por una encuesta a través de Internet son similares a los recogidos por una encuesta presencial, planteando como hipótesis que existe equivalen- 
cia en el tipo de población que coopera en cada modo y en las preguntas de la encuesta.

Las grandes diferencias entre la población general y los usuarios de Internet impiden aceptar la primera parte de la hipótesis, puesto que las encuestas a través de la red presentan un perfil más joven y con mayor nivel educativo. La práctica totalidad de las investigaciones analizadas presentan diferencia en las variables objeto de la investigación, según se haya respondido el cuestionario frente a un entrevistador o en el ordenador del entrevistado.

La revisión de la literatura impide aceptar que los resultados de la aplicación de un cuestionario sean los mismos independientemente de la modalidad utilizada en su aplicación. Es importante considerar esta afirmación con precaución, puesto que, en este momento de baja tasa de colaboración en las encuestas y de tremenda segmentación en la forma de acceder a cada colectivo, es posible que la encuesta por Internet consiga información de personas que nunca participan en encuestas presenciales y telefónicas, lo que explicaría las diferencias localizadas en las respuestas (De Leeuw et al., 2008; Link y Mokdad, 2006). De hecho, hay numerosos trabajos que recomiendan la utilización de encuestas en Internet como un complemento al resto de modalidades (Fricker y Schonlau, 2002; Ilieva et al., 2002; Schneider et al., 2005; Converse et al., 2008; Manfreda y Vehovar, 2008; Holmberg, 2010; Revilla, 2010). La relativa «novedad» de las encuestas por Internet precisa realizar más investigación sobre el tema.

\section{Referencias bibliográficas}

ADAMS, R. (2011). «Aprovechar los medios de comunicación social para la captación e involucración del actual panelista on-line». Investigación y Marketing, 110, 54-55.

AKL, E.A.; MARroun, N.; Klocke, R.A.; Montori, V. y SCHUNEMANN, H.J. (2005). «Electronic mail was not better than postal mail for surveying residents and faculty». Journal of Clinical Epidemiology, 58, 425-429.

ÁlvareZ, B.; ÁlVAREZ, T. y MolPeCERES, B. (2005). «Aceptación social de la encuestas en Internet: Desarrollo de una aplicación específica». Metodología de Encuestas, 7, 5-17.

ANEIMO (2010). «Nuevos datos sobre el sector de la investigación de mercados en 2009». Investigación y Marketing [en línea], 108, 108-109. <http://www.aedemo. es/aedemo/images/stories/varios/datos_sector_2009_aedemo.pdf>.

AQUiLINO, W.S. (1994). «Interview Mode Effects in Survey of Drug and Alcohol Use». Public Opinion Quarterly, 58, 210-240.

Asociación para la InVESTigaCión de los Medios de Comunicación (AIMC) (2009). Audiencia en Internet según el EGM, febrero-marzo de 2008. Acceso: 25 de mayo de 2009.

BAKER, R. et al. (2010). «Research synthesis: AAPOR Report on online panels». Public Opinion Quarterly, 74, 711-781.

BANDILlA, W.; BOSNJAK, M. y ALTDORFER, P. (2003). «Survey administration effects?: A comparison of web-based and traditional written selfadministered surveys using ISSP environment module». Social Science Computer Review, 21, 235-243.

BECH, M. y Bo KRISTENSEN, M. (2009). "Differential response rates in postal and web-based surveys among older respondents». European Survey Research Association, $3(1), 1-6$. 
Bethlehem, J. y Stoop, I. (2007). Online panels: A paradigm theft? Ponencia presentada en la reunión anual de la Associacion for Survey Computing, Southampton, Inglaterra.

Biemer, P.P. y LyberG, L.E. (2003). Introduction to Survey Quality. Nueva York: Wiley.

BiRNHOLTZ, J. et al. (2004). "Cash, electronic, and paper gift certificates as respondent incentives for a web-based survey of technologically sophisticated respondents». Social Science Computer Review, 22, 355-362.

Blasius, J. y BRAndT, M. (2010). «Representativeness in Online Surveys through Stratified Samples». Bulletin de Methodologie Sociologique, 107, 5-21.

Bonke, J. y FAllesen, P. (2010). "The impact of incentives and interview method on response quantity and quality in diary — and booklet — based surveys». Survey Research Methods, 4, 91-101.

BONSJAK, M. y TuTEN, T.L. (2003). «Prepaid and promised incentives in web surveys». Social Science Computer, 21, 208-217.

Bradley, N. (1999). "Sampling for Internet Surveys: An Examination of respondent Selection for Internet Research». International Journal of Market Research, 41, 387-395.

CATANiA, Josep A. et al. (1996). "Effects of Interviewer Gender, Interviewer Choice, and Item Wording of Responses to Questions Concerning Sexual Behavior». Public Opinion Quarterly, 60, 345-375.

Centro de InVESTIGACIONES SOCIOlÓGICAS (2007a). La percepción social de las encuestas. Estudio número 2.676, febrero.

- (2007b). La percepción social de las encuestas: Ficha técnica [en línea]. Estudio número 2.676, febrero. <http://www.cis.es/cis/opencms/-Archivos/Marginales/2660_2679/2676/Es2676_Ftecnica_enlace.html> [Consulta: 6 noviembre 2010].

ChANG, L. y KrosnicK, J.A. (2009). «National surveys via RDD telephone interviewing versus the Internet: comparing sample representativeness and response quality». Public Opinion Quarterly, 73, 641-678.

- (2010). «Comparing Oral Interviewing with Self-Administered Computerized Questionnaires: An Experiment». Public Opinion Quarterly, 74, 154-167.

Christian, L.M.; Dillman, D.A. y SmyTh, J.D. (2008). "The effects of mode and format on answers to scalar questions in telephone and web surveys». En: LEPKOWSKI, J.M.; TuCKer. C.; Brick. J.M.; De LeEUW, E.D.; JAPEC, L.; LAVRAKAS, P.J.; LINK, M.W. y SANGSTER, R.L. (eds.). Advances in telephone survey methodology. Nueva York: Wiley, 250-275.

Cobanoglu, C.; Moreo, P.J. y Warde, B. (2001). "A comparison of mail, fax and web-based survey methods». International Journal of Market Research, 43, 441-452.

Converse, P. et al. (2008). «Response Rates for Mixed-Mode Surveys Using Mail and E-mail/Web». American Journal of Evaluation, 29, 99-107.

Cook, C. et al. (2000). «A Meta-Analysis of Response Rates in Web- or Internet Based Surveys». Educational and Psychological Measurement, 60, 821-836.

Couper, M.P. (2000). «Web surveys: A review of issues and approaches». Survey Public Opinion Quarterly, 64, 464-494.

- (2008). Designing effective web surveys. Nueva York: Cambridge University Press.

Couper, M.P.; Blair, J. y Triplett, T. (1999). A Comparison of Mail and E-Mail for a Survey of Employees in Federal Statistical Agencies. Universidad de Maryland. Colección Current Research Papers del Survey Research Center. 
Couper, M.P.; Tourangeau, R. y Marvin, T. (2009). «Taking the Audio Out of Audio-CASI». Public Opinion Quarterly, 73, 281-303.

Couper, M.P. y Miller, P.V. (2009). «Web surveys methods: Introduction». Public Opinion Quarterly, 72 (5), 831-835.

COUPER, M. et al. (2004). «Does voice matter? An interactive voice response (IVR) experiment». Journal of Official Statistics, 20 (3), 551-570.

CuENDE, D. (2010). "Visión global del mercado de la investigación - ESOMAR 2009». Investigación y Marketing, 106, 52-53.

Dannetun, Eleanor; Tegnell, Alain y Gieseche, Jean (2007). Parents' attitudes towards hepatitis $B$ vaccination for their children: A survey comparing paper and web questionnaires. Suiza: Bmc Public Health.

DAVIDOV, E. y DEPNER, F. (2009). «Testing for measurement equivalence of human values across online and paper and pencil surveys». Quality and Quantity [en línea]. <http://www.springerlink.com/content/1072323173u57841/?p=7b6fbca53b604 b35ae26c442ba436422\&pi=0> [Consulta: 10 junio 2010].

Delavande, A. y RoHWEDDER, S. (2009). «Elicting subjective probabilies in internet surveys». Public Opinion Quarterly, 72 (5), 866-891.

De Leeuw, E.D. (1992). Data Quality in Mail, Telephone and Face to Face Surveys. Amsterdam: TT-Publikaties.

- (2008). «Choosing the method of data collection». En: DE LEEUW, E.D.;

De Leeuw, E.; Dillman, D.A. y Hox, J.J. (2008). «Mixed mode surveys: When and why». En: De Leeuw, E.D.; Hox, J.J. y Dillman, D.A. (eds.). International Handbook of Survey Methodology. Nueva York: Lawrence Erlbaum Associates y Asociación Europea de Metodología, 299-316.

De Leeuw, E. y VAn der Zouwen, J. (1988). "Data quality in telephone and face to face surveys: a comparative meta-analysis». En: Groves, R.M.; BIEMER, P.P.; Lyberg, L.E.; Massey, J.T.; Nichols II, W.L. y Waksberg, J. Telephone Survey Methodology. Nueva York: Wiley, 283-298.

DÍAZ DE RADA, V. (2010). "Eficacia de las encuestas por internet: un estudio preliminar». Revista Española de Sociología, 13, 49-79.

- (2011). «Encuestas con encuestador y autoadministradas por internet. ¿Proporcionan resultados comparables?». Revista Española de Investigaciones Sociológicas, 136, 49-90.

Díaz DE RADA, V. y NúNEzZ Villuendas, A. (2008). Estudio de las incidencias en la investigación mediante encuesta. Madrid: Centro de Investigaciones Sociológicas.

Dillman, D.A. (1991). "The Design and Administration of Mail Surveys». Annual Review of Sociology, 17, 225-249.

- (2000). Mail and Internet Survey. Nueva York: Willey.

Dillman, Don A. et al. (2001). Response rate and measurement differences in mixed-mode surveys using mail, telephone, interactive voice response (IVR) and the Internet. Ponencia presentada en la 56. ${ }^{\mathrm{a}}$ conferencia anual de la Asociación Americana de Opinión Pública (American Association for Public Opinion Research-AAPOR).

Dillman, D.A.; SMYTH, J. y Christian, L.M. (2009). Internet, Mail and Mixed-Mode Surveys: The Tailored Design Method. Nueva York: John Wiley.

Diment, K. y GarretT-JoneS, S. (2007). «How Demographic Characteristics Affect Mode Preference in a Postal/Web Mixed-Mode Survey of Australian Researchers». Social Science Computer Review, 25, 410-417.

Duffy, B.; Terhanian, G.; Bremer, J. y SMith, K. (2005). «Comparing data from online and face-to-face surveys». International Journal of Market Research, 47, 615-639. 
Durrant, G.B. et al. (2010). «Effects of Interviewer Attitudes and Behaviors on Refusal in Household Surveys». Public Opinion Quarterly, 74, 1-36.

DyKeMA, J.; BASSON, D. y SCHAEFFER, N.C. (2008). «Face to face surveys». En: DONSBaCH, W. y TraugotT, M.W. (eds.). The SAGE Handbook of Public Opinion Research. Thousand-Oaks, LA: Sage, 240-248.

Erdos, P.L. (1970). Professional Mail Surveys. Nueva York: McGraw Hill. Edición original 1951.

Europe Internet Usage Stats and Population Statistics (2011). Internet World Stats [en línea]. <http://www.internetworldstats.com/stats4.htm> [Consulta: 15 junio 2009].

FERnÁNDEZ AEDÉval, M. (2006). «La población internauta en España, 2005». Investigación y Marketing, 91, junio, 5-10.

Fowler, F.J. y MANGLIONE, T.W. (1990). Standarized survey interviewing. Newbury Park, CA: Sage.

Fox, J. et al. (2003). «Conducting research using web-based questionnaires: practical, methodological and ethical considerations». International Journal of Social Research Methodology, 6, 167-180.

Fricker, S. et al. (2005). "An Experimental Comparison of Web and Telephone Surveys». Public Opinion Quarterly, 69, 370-392.

Fricker, R. y SCHONLAU, M. (2002). "Advantages and disadvantages of internet research surveys: evidence tom the literature». Field Methods, 14, 347-367.

Fricker, S.; Galesic, M.; Tourangeau, R. y Yan, T. (2005). «An Experimental Comparison of Web and Telephone Surveys». Public Opinion Quarterly, 69 (3), 370-392.

Fundación Telefónica (2011). La sociedad de la información en España 2010. Madrid: Fundación Telefónica.

GIBSON, L. (2010). Using e-mail interviews [en línea]. <http://www.socialsciences. manchester. ac.uk/realities/resources/toolkits/email-interviews/09-toolkit-emailinterviews.pdf> [Consulta: 6 julio 2010].

GÖrTIZ, Anja S. (2006). "Incentives in web studies: methodological issues and a review». International Journal of Internet Science, 1, 58-70.

- (2008). «The Long-Term Effect of Material Incentives on Participation in Online Panels». Field Methods, 20, 211-225.

Goyder, J. (1987). The Silent Minority, Nonrespondents on Sample Surveys. Cambridge: Polity Press.

Goyder, J. y LEIPER, J.M. (1985). «The decline in survey response: A social values interpretation». Sociology, 19, 55-71.

GrAFT, L. (2002). «Assesing internet questionnaires: the online pretest lab». En: Batinic, B.; Reips, U.D.; Bonja, M. y Werner, A. (eds.). Online Social Sciences. Seattle: Hogrefe and Huber.

Groves, R.M. (1989). Survey Error and Survey Cost. Nueva York: Wiley.

Groves, R.M. y COUPER, M.P. (1998). Nonresponse en Household Interview Surveys. Nueva York: Wiley.

Groves, R.M.; Fowler, F.J.; COUPer, M.P.; LePKOwski, J.M.; Singer, E. y TOURANGEAU, R. (2009). Survey Methodology. Nueva York: Wiley.

Groves, R.M. y KaHn, R. (1979). Surveys by telephone. Nueva York: Academic Press.

Harmon, T.; Turner, Ch.F.; Rogers, S.M.; Eggleston, E.; Roman, A.M.; Villarroel, M.A.; Chromy, J.R.; Ganapathi, L. y Sheping Li, S. (2009). «Impact 
of T-ACASI on Survey Measurements of Subjective Phenomena». Public Opinion Quarterly, 73, 255-280.

HEBERLEIN, T. y BAUMGARTNER, R. (1978). «Factors affecting response rates to mailed questionnaires: A quantitative analysis of the published literature». American Sociological Review, 43, 447-462.

- (1981). "Is a questionnaire necessary in a second mailing?». Public Opinion Quarterly, 45, 102-108.

Heerwegh, D. (2005). «Effects of Personal Salutations in E-mail Invitations to Participate in a Web Survey». Public Opinion Quarterly, 69, 588-598.

- (2009). «Mode differences between face-to-face and web surveys: An experimental investigation of data quality and social desirability effects». International Journal of Public Opinion Research, 21, 111-120.

HeERweGH, D. y LoOsveldt, G. (2009). «Face to face versus web surveying in a high internet coverage population». Public Opinion Quarterly, 72 (5), 836-846.

- (2009). "Explaining the intention to participate in a web survey: a test of the theory of planned behavior». International Journal of Social Research Methodology, $12,181-195$.

Hoek, J.; Guendall, P. y Healey, B. (2002). «Web based polling: An evaluation of survey modes». Australasian Journal of Market Research, 10, 23-34.

HolBroOK, A.L. (2003). «Telephone versus face-to-face interviewing of national probability samples with long questionnaires». Public Opinion Quarterly, 67, 79-125.

Holmberg, A. et al. (2010). "Contact Strategies to Improve Participation via the Web in a Mixed-Mode Mail and Web Survey». Journal of Official Statistics, 26, 3, 465-480.

HoOgendoorn, A.W. y DAALmans, J. (2009). "Nonresponse in the recruitment of an internet panel based on probability sampling». Survey Research Methods, 3 (2), 59-72.

Hox, J.J. y Dillman, D.A. (eds.). International Handbook of Survey Methodology. Nueva York: Lawrence Erlbaum Associates y Asociación Europea de Metodología, 113-135.

Ilieva, J.; Baron, S. y HeAley, N.M. (2002). "Online surveys in marketing research». International Journal of Market Research, 44, 361-376.

Instituto DE ESTUdios AVANZADOS DEL CONSEJO Superior DE INVESTIGACIONES CIENTÍFICAS IESA-CSIC (2010). La situación de los andaluces en el extranjero.

Instituto Nacional de Estadística (2009). Encuesta de Tecnologías de la Información en los Hogares [en línea]. Madrid: INE. <www.ine.es>.

INTERNET WORLD STATS (2011). Internet Usage in Europe (junio 2010) [en línea]. <http://www.internetworldstats.com/stats4.htm\#europe> [Consulta: 22 marzo 2011].

Jacobs, M.; Cross, J. y SMAILES, E. (1994). «CIM: Computer Interviewing by Mail». Quality and Quantity, 28, 137-150.

JAPEC, L. (2006). "Quality Issues in Interview Surveys». Bulletin de Methodologie Sociologique, 90.

JONES, R. y PRITT, N. (1999). «Health surveys in the workplace: comparison of postal, email and World Wide Web methods». Occupational Medicine-Oxford, 49, 556-558.

KalsbeeK, W.D. y Agans, R.B. (2008). «Sampling and weighting in household telephone surveys». En: LePKOWSKI, J.M.; TUCKER, C.; BRICK, J.M.; De LeEUW, E.D.; JaPeC, L.; LAVRaKas, P.J.; LinK, M.W. y SANGSTER, R.L (eds.). Advances in telephone survey methodology. Nueva York: Wiley, 250-275. 
Kalton, G. (2000). «Developments in survey research in the past 25 years». Survey Methodology, 26 (1), 3-10.

Kaplowitz, M.D.; Hadlock, T.D. y Levine, R.S. (2004). "A Comparison of Web and Mail Survey Response Rates». Public Opinion Quarterly, 68 (2), 94-101.

KENT, R. y LeE, M. (1999). "Using the Internet for Market Research: A Study of Private Trading on the Internet». International Journal of Market Research, 41, 377-385.

KisH, L. (1965). Survey Sampling. Nueva York: Wiley.

KörmENDI, E. (1988). "The quality of income information in telephone and face to face surveys». En: Groves, R.M.; Biemer, P.P.; Lyberg, L.E.; MASseY, J.T.; Nichols II, W.L. y WaKsberg, J. Telephone Survey Methodology. Nueva York: Wiley, 283-298.

Kreuter, F.; Presser, S. y Toureangeau, R. (2008). "Social desirability bias in CATI, IVR and Web Surveys». Public Opinion Quarterly, 72 (5), 847-865.

KROSNICK, J.A. (1991). "Response strategies for coping with the cognitive demands of attitude measures in surveys». Applied Cognitive Psychology, 5, 213-236.

- (1999). «Survey research». Annual Review of Psychology, 50, 537-567.

KWAK, N. y RADler, B. (2002). "A Comparison between mail and web surveys: response pattern, respondent profile and data quality». Journal of Official Statistics, 18 (2), 257-273.

LAMAS, C. (2007). The Testing Web interviewing in the EGM Multimedia Survey. 2007 EMRO Conference.

«La mitad de los españoles se conectó a Internet en el último mes». Investiga, 29 (verano 2010), 4.

«Las mujeres duplican su presencia en la red en los últimos diez años». Investiga, 25 (2009), 6 .

LINK, M.W. y MOKDAD, A. (2006). "Can web and mail survey modes improve participation in a RDD-based National Health Surveillance?». Journal of Official Statistics, 22 (2), 293-312.

Llauradó, O. (2006). «El trabajo de campo Online: Qué hemos aprendido en los últimos diez años». Investigación y Marketing, 91, junio, 25-40.

LÓPEZ, A. (2006). "Que las escasas diferencias no nos impidan aprovechar las ventajas de Access Panel Online». Investigación y Marketing, 91, junio, 20-24.

- (2008). "¿Seguimos hablando del online». Investigación y Marketing, 98, marzo, 28-30.

Manfreda, K.L. y Vehovar, V. (2008). «Internet surveys». En: De LeEuw, E.D.; Hox, J.J. y Dillman, D.A. (eds.). International Handbook of Survey Methodology. Nueva York: Lawrence Erlbaum Associates y Asociación Europea de Metodología, 264-284.

Manfreda, K.L.; BosnjaK, M.; BerzelaK, J.; HaAs, I. y Vehovar, V. (2008). «Web surveys versus other survey modes: a meta-analysis comparing response rates». International Journal of Market Research, 50 (1), 79-104.

MANZANO, V. y ANDREU, J. (2000). «Formatos para ítems en las encuestas electrónicas: Antecedentes y propuestas». Metodología de Encuestas, 2, 61-101.

Manzano, V.; LozAnO, O. y FernándeZ Prados, J.S. (2003). «Medidas subjetivas para formatos de respuesta en encuestas informatizadas». Metodología de Encuestas, 5, 213-226.

Martín, Montserrat y Moré, David (2010). «Las claves de la investigación on line». Investiga, 9 (verano), 6. 
McCullough, D. (1998). "Web-based market research ushers in new age». Marketing News, 32 (9), 27-28.

McDonald, H. y ADAm, S. (2003). "A comparison of online and postal data collection methods in marketing research». Marketing Intelligence and Planning, 21, 85-95.

Metha, R. y Sivadas, E. (1995). «Comparing Response Rates and Response Content in Mail versus Electronic Mail Surveys». Journal of the Market Research Society, 37, 429-439.

MuÑOZ LEIVA, F. et al. (2009). «Improving the response rate and quality in web-based surveys through the personalization and frequency of reminder mailings». Quality and Quantity [en línea]. <http://www.springerlink.com/content/3r5h867076046 $\mathrm{q} 7 \mathrm{x} /$ ?p=70a43be4413c4fb89ad6d4f48df38b48\&pi=2> [Consulta: 10 junio 2010].

NiCOlaAs, Gerry; KerT, Thomson y Lynn, Peter (2000). The feasibility of conducting Electoral Surveys in the UK by telephone. Londres: National Centre for Social Research.

ObSERVATORIo Nacional de las Telecomunicaciones y la SOCIEDAD DE la INFORMACIÓN (2010). La sociedad en Red 2009: Informe Anual de la Sociedad de la Información en España 2009 (edición 2010) [en línea]. <http://www.red.es/media/ registrados/2010-09/1285751492965.pdf?aceptacion $=6190 \mathrm{fd} 08 \mathrm{c} 92191 \mathrm{f} 639 \mathrm{bla0}$ 44a406db6e> [Consulta: 22 marzo 2011].

Pasadas del Amo, S.; Trujillo Carmona, Manuel; Sánchez Valenciano, Ana y CAÑADAS RECHE, Luis (2011). «La incorporación de las líneas móviles al marco muestral de las encuestas telefónicas: Pertinencia, métodos y resultados». Metodología de Encuestas, 13, 33-54.

Pasadas del Amo, S.; Soria Zambrano, M. y Zarco Uribe-Echevarría, M. (2006). "¿importa el prefijo?: Una aproximación a las diferencias territoriales en las pautas de respuesta a las encuestas telefónicas». Metodología de Encuestas, 7 (3), 3-12.

PedrazA, P. de et al. (2010). «A Spanish Continuous Volunteer Web Survey: Sample Bias, Weighting and Efficiency». Revista Española de Investigaciones Sociológicas, 131, 109-130.

Peleteiro, I. y Gabardo, J. (2006). «Los hogares “exclusivamente móviles” en la investigación telefónica de audiencia». Metodología de Encuestas, 7 (3), 17-40.

Porter, S.R. y Whitcomb, M.E. (2006). "Mixed-mode contacts in web surveys: paper is not necessary better». Public Opinion Quarterly, 71, 635-648.

Pratesi, M.; Manfreda, K.L.; Biffignandi, S. y Vehovar, V. (2004). «List-based Web Surveys: Quality, Timeliness and Nonresponse in the Steps of the Participation Flow». Journal of Official Statistics, 20 (3), 451-465.

Preece, M.J. et al. (2010). "Lottery incentives and online surveys response rates». Survey Practice [en línea]. <www.surveypractice.org> [Consulta: 19 enero 2011].

Presser, S. et al. (2004). Questionnaire Development Evaluation and Testing Methods. Nueva York: Wiley.

RAO, K. et al. (2010). «Recruiting probability samples for a multi-mode research panel with internet and mail components». Public Opinion Quarterly, 74, 68-84.

Revilla, M. (2010). "Quality in Unimode and Mixed-Mode designs: A MultitraitMultimethod approach». Survey Research Methods, 4, 151-164.

Rodríguez Esteban, M. (2008). Industria Española de Investigación de Mercados y Opinión Pública en 2007 [en línea]. Barcelona: AEDEMO. <www.aedemo.es> [Consulta: 30 noviembre 2009]. 
Roster, C.A.; Rogers, R.D.; Albaum, G. y Klein, D. (2004). «A comparison of response characteristics from web and telephone surveys». International Journal of Market Research, 46 (3), 359-373.

SAltZMAn, A. (1993). «Improving Response rates in Disk-By-Mail Surveys». Marketing Research, 5, 32-39.

SchneIDER, Sid J. et al. (2005). «Telephone, Internet, and Paper Data Collection Modes for the Census 2000 Short Form». Journal of Official Statistics, 21, 89-101.

SCHRÄPLER, J.P. et al. (2010). "Changing from PAPI to CAPI: Introducing CAPI in a Longitudinal Study». Journal of Official Statistics, 26 (2), 239-269.

SCHONLAU, M. et al. (2003). "A comparison between responses from a propensity-weighted web survey and an identical RDD survey». Social Science Computer Review, 21, 1-11.

- (2010). "Collecting Genetic Samples in Population Wide (Panel) Surveys: Feasibility, Nonresponse and Selectivity». Survey Research Methods, 4, 121-126.

SHIH, T.H. y FAN, X. (2008). "Comparing Response Rates from Web and Mail Surveys: A Meta-Analysis». Field Methods, 20, 249-271.

SINGER, E. (2004). "Confidentiality, risk perception, and survey participation». Chance, 17, 30-34.

- 2006). "Special issue: nonresponse bias in household surveys», Public Opinion Quarterly, 70 (5), 637-645.

Singer, E. y Presser, S. (2008). «Privacy, confidentiality and response burden as factors in telephone survey response». En: LEPKOWSKI, J.M.; TUCKER. C.; BRICK. J.M.; De LeeUw, E.D.; JapeC, L.; Lavrakas, P.J.; LinK, M.W. y SANGSTER, R.L (eds.). Advances in telephone survey methodology. Nueva York: Wiley, 449-470.

Singer, E.; Van HoewyK, J. y Neugebauer, R. (2003). "Attitudes and behavior: the impact of privacy and confidentiality concerns on participation in the 2000 census». Public Opinion Quarterly, 65, 368-384.

SKIM SOFTwARE DiviSION (1996). «Disk-by-Mail and Disk-by-Mail benefist». SKIM Software Division Newsletter, otoño, 3.

Sparrow, N. y CURTICE, J. (2004). "Measuring the attitudes of the general public via internet polls: An evaluation». International Journal of Market Research, 46 (1), 23-44.

SteeH, C. y Piekarski, L. (2008). «Accommodation new technologies: mobile and VoIP communication». En: LePKOwski, J.M.; TuCKer. C.; Brick. J.M.; De Leeuw, E.D.; Japec, L.; Lavrakas, P.J.; Link, M.W. y Sangster, R.L (eds). Advances in telephone survey methodology. Nueva York: Wiley, 423-448.

STOOP, I. (2006). The hunt for the last respondent. Nonresponse in sample surveys. The Hague: Social and Cultural Planning Office of the Netherlands.

STOOP, I. et al. (2010). Improving Survey Response: lessons learned from the European Social Survey. Nueva York: Wiley.

SYKES, W. y COLLINS, M. (1988). «Efects of mode of Interviewing: experiments in the UK». En: Groves, R.M.; Biemer, P.P.; LyberG, L.E.; MASSEY, J.T.; Nichols II, W.L. y Waksberg, J. Telephone Survey Methodology. Nueva York: Wiley, 301-320.

TAYLOR, H. (2000). "Does internet research work?: Comparing online survey results with telephone survey». International Journal of Market Research, 41, 51-63.

Thomas, R.K. y KLeIN, J.D. (2006). «Merely Incidental?: Effects of Response Format on Self-reported Behavior». Journal of Official Statistics, 22, 221-244.

Toepoel, V.; Das, M. y Van SOest, V. (2009). «Effects of design in web surveys». Public Opinion Quarterly, 72 (5), 985-1007. 
Tourangeau, R. (2004). «Survey Research and societal change». Annual Review of Psychology, 55, 775-801.

Tourangeau, R.; Couper, M.P. y Conrad, F. (2007). "Color, labels, and interpretative heuristic for response scales». Public Opinion Quarterly, 71, 91-112.

Tourangeau, R.; Rips, L.J. y Rasinski, K. (2000). Psychology of Survey Response. Cambridge: Cambridge University Press.

Tourangeau, R. y Smith, T. (1996). «Asking Sensitive Questions: The impact of Data Collection, Question Format, and Question Context». Public Opinion Quarterly, 60, 275-304.

TSE, A.C.B. (1995). "Comparing two methods of sending out questionnaires: e-mail versus mail». International Journal of Market Research, 37.

- (1998). «Comparing Response Rate, Response Speed and Response Quality of Two Methods of Sending Questionnaires: E-mail vs. Mail». International Journal of Market Research, 40.

TuCKer, C. y LePKOWSKI, J.M. (2008). «Telephone survey methods: Adapting to change». En: LePKOWSKI, J.M.; TuCKer. C.; BricK. J.M.; De LeEUW, E.D.; JAPEC, L.; Lavrakas, P.J.; Link, M.W. y Sangster, R.L. (eds.). Advances in telephone survey methodology. Nueva York: Wiley, 3-26.

VAlLS, O. (2007). «Investigación on-line: menos costes y mayor rapidez». Investiga: Publicación de Investigación de Mercados, 17, verano, 24-28.

VAN DEN BRAKel, J.A. (2006). "An Experiment with Data Collection Modes and Incentives in the Dutch Family and Fertility Survey for Young Moroccans and Turks». Field Methods, 18, 321-334.

Vehovar, V.; Manfreda, K.L. y Koren, G. (2008). «Internet surveys». En: DonsBaCH, W. y TraugotT, M.W. (eds.). The SAGE Handbook of Public Opinion Research. Thousand-Oaks, CA: Sage, 271-283.

Verge Mestre, T. (2009). "Deseabilidad social y género: el efecto del sexo del entrevistador (a) sobre las respuestas del encuetado (a)», Metodología de Encuestas, $11,27-45$.

WEISBERG, H.F. (2005). The total survey error approach: A guide for the new science of survey research. Chicago: University of Chicago Press.

Wert, J.I. (2000). "La encuesta telefónica». En: GARCÍA FERrando, M.; IbáÑEZ, J. y Alvira, F. (eds.). El Análisis de la Realidad Social. 3a ed. Madrid: Alianza, 203217. Edición original: 1986.

Willmes, P. y Oosterveld, P. (2003). «The best of both worlds». Marketing Research, 23-26.

Yang, Yongwei; Callegaro, Mario; Bhola, Dennison S. y Dillman, Don A. (2011). "IVR and web administration in structured interviews utilizing rating scales: exploring the role of motivation as a moderator to mode». International Journal of Social Research Methodology, 14, 1-5.

Zorrilla, Á. (2009). "Nuestras tasas de respuesta son del 40\%, mientras que el sector se sitúa en un 5\%", German Loewe, director de Netquest». Investiga, 27 (invierno), 18.

- (2010). «El poder de la investigación online». Investiga, 29, verano, 14. 


\section{Anexo. Características técnicas de la investigación}

\section{La situación de los andaluces en el extranjero}

Realizada por el Instituto de Estudios Sociales Avanzados del Consejo Superior de Investigaciones Científicas (IESA-CSIC) y financiada por la Consejería de Gobernación de la Junta de Andalucía

(Fuente: reproducido de Instituto de Estudios Avanzados del Consejo Superior de Investigaciones Científicas-IESA, 2010)

Universo: personas residentes en el extranjero, empadronadas en Andalucía con edades iguales o superiores a 18 años.

Tamaño de la muestra: 2.446 entrevistas.

Tipo de entrevista: nominal a través de un modelo mixto de administración del cuestionario.

Canal principal (recepción del cuestionario): cuestionario postal autoadministrado.

Canales secundarios (alternativas ofrecidas al entrevistado una vez le llega el cuestionario en papel): cuestionario digital autoadministrado, así como entrevista telefónica asistida por ordenador $(\mathrm{CATI})^{27}$.

Tipo de muestreo: muestreo bietápico, estratificado por conglomerados, y elección en cada conglomerado, por muestreo aleatorio simple, de 10 unidades por cada unidad muestral teórica.

Estratificacion: conjuntos geopolíticos que agrupan a países con condiciones homogéneas de desarrollo socioeconómico y próximas geográficamente. Unión Europea, Europa desarrollada no UE, América Central y del Sur, Norteamérica,

27. Reproducimos aquí el texto en el que se explicaba la forma de respuesta:

«El cuestionario ha sido elaborado para que usted pueda completarlo fácilmente rellenando las diferentes casillas. Puede participar en la encuesta a través de las siguientes vías:

- Correo postal con franqueo pagado. Cumplimente el cuestionario que acompaña a esta carta y envíelo en el sobre de respuesta dispuesto al efecto. Se trata de un envío gratuito sin ningún coste para usted.

- Internet. Accediendo al sitio de Internet 'www.nombre del sitio', a través de la aplicación informática dispuesta a tal efecto, para lo cual deberá identificarse con el código de acceso al cuestionario que aparece en rojo en el encabezado de esta carta.

- Teléfono. Si desea contestar a la encuesta por teléfono, háganos llegar un número de teléfono de contacto y las horas en las que podemos localizarle. Una operadora se pondrá en contacto con usted lo antes posible para hacerle las preguntas del cuestionario. Puede hacer esto a través de una de las siguientes vías:

a) Enviando la solapa de esta carta con sus datos de contacto en el sobre de respuesta adjunto.

b) Accediendo al sitio de Internet: "www.nombre del sitio" y completando la información de contacto telefónico.

c) Llamando al número de teléfono (número de teléfono) podrá grabar en el buzón su código de acceso al cuestionario 'COD_ID’ y sus datos de contacto telefónico (número y horas de contacto).

Le rogamos que responda a esta encuesta tan pronto [...]».

Fuente: reproducido de IESA, 2010. 
Oceanía y Norte de África. Se han excluido los conjuntos que representan un porcentaje equivalente o inferior al 0,3\% de los andaluces en el exterior.

Conglomerados y elección de la unidad final de muestreo: se han seleccionado los países con población mayor o igual a 125 andaluces en el exterior y cuya muestra proporcional sea superior a 25 personas. Para el resto de países, de forma proporcional al número de andaluces hasta completar la muestra de cada conjunto geopolítico. Las unidades finales a muestrear son las personas residentes en los 20 países seleccionados.

Niveles de error máximo de muestreo: se calcula un 2\% como nivel de error de referencia, bajo la hipótesis de que se cubre la muestra teórica.

Fecha de realización: de septiembre de 2009 a mayo de 2010. 